\title{
Riesgos operacionales: Reto actual en las empresas de operaciones de guaya fina
}

Operational risks: Current challenge in the operating companies of guaya fina

\section{Leonela Matos}

matoslb22@gmail.com

Código ORCID: 0000-0001-8462-2066

\section{Daniela Matos}

danielamatos86@gmail.com

Código ORCID: 0000-0003-3607-4397

Universidad del Zulia, Núcleo Costa Oriental del Lago

Articulo recibido en julio 2019 Arbitrado en agosto 2019 Publicado en enero 2020

\section{Resumen}

Identificar qué peligros acechan la seguridad del trabajador donde quiera que se encuentre en el lugar de trabajo es hoy en día un reto para las organizaciones, desde esta perspectiva el propósito de este artículo es determinar los riesgos operaciones como un mecanismo para asegurar el cumplimiento de las normas y procedimientos de seguridad, higiene y ambiente. A tal fin se consideró realizar un análisis en el contexto de las empresas que están orientadas a ejecutar actividades de subsuelo sin sacar la tubería de producción, conocidas como operaciones de guaya fina, específicamente las ubicadas en la Costa Oriental del Lago de Maracaibo, Venezuela. Según los resultados estas empresas, no están gestionando de manera acertada la prevención de riesgos.

Palabras clave: Guaya fina, prevención de riesgos, riesgos operaciones, seguridad del trabajador, seguridad, higiene y ambiente

\section{Abstract}

Identifying what hazards threaten the safety of the worker wherever he is in the workplace is today a challenge for organizations, from this perspective the purpose of this article is to determine the operational risks as a mechanism to ensure compliance with the standards and procedures for safety, hygiene and environment. To this end, an analysis was considered in the context of companies that are oriented to carry out subsoil activities without removing the production pipeline, known as fine guaya operations, specifically those located on the eastern coast of Lake Maracaibo, Venezuela. According to the results, these companies are not managing risk prevention correctly. 
Keywords: Fine guava, risk prevention, operational risks, worker safety, safety, hygiene and environment

\section{INTRODUCCIÓN}

En el campo de las empresas petroleras, se encuentran las operaciones de subsuelo con guaya fina, las cuales son actividades que se realizan con el fin de colocar equipos y herramientas en el pozo, para permitir la producción de los fluidos del mismo en forma eficiente y segura, minimizando o anulando los posibles riesgos. Cabe destacar que, las operaciones que se ejecutan con guaya fina, deben garantizar la competitividad e implantar sistemas que les permitan gestionar los riesgos a los cuales se encuentran expuestos sus trabajadores.

Es por ello que, la formación en materia de inseguridad, en los diferentes puestos de trabajo y en la función de cada empleado, se debe realizar y adaptar a la evaluación de los riesgos, repitiéndose periódicamente para así evitar el daño profesional (Menéndez, 2008). De esta forma, la seguridad en el compromiso se constituye en una disciplina que tiene como misión el estudio de los acontecimientos laborales, analizando los factores de peligros y las causas que producen los accidentes de trabajo.

Dentro de este orden, la seguridad industrial juega un papel importante puesto que es un conjunto de principios, leyes, criterios y normas, cuyo objetivo es prevenir accidentes así como controlar los peligros que pueden ocasionar daños a personas, medio ambiente equipos y materiales. En efecto, el principal objetivo de la higiene industrial es la aplicación de medidas adecuadas para prevenir y controlar riesgos en el medio ambiente de trabajo, entendiéndose éstos como riesgos operacionales.

Para Ruiz (2007) los riesgos en las operaciones son los diversos peligros o factores de riesgos que pueden causar daños a la salud, malestar e ineficiencia significativa en los trabajadores en cada ámbito del área de trabajo que se desempeñan. En este sentido, para realizar actividades conjuntas de las diferentes disciplinas, según este autor, se hace necesario describir la siguiente clasificación: riesgos físicos, riesgos químicos, riesgos biológicos y riesgos disergonómicos.

Mientras, para Hualde (2010), los riesgos operacionales son situaciones posibles que puedan reducir los ingresos de la empresa o puedan erosionar la imagen ocasionando una pérdida operacional futura, lo que es tratada como riesgo en las operaciones. Para este autor, es importante, entonces, reconocer que todas las actividades desarrolladas por la empresa $u$ organización conllevan a un riesgo operativo, el cual debe ser gestionado y medido para que el negocio se siga desarrollando de una manera óptima.

Bajo estas consideraciones, las investigadoras consideran los riesgos operacionales como aquellas situaciones o condiciones de trabajo que pueden 
perjudicar la salud del trabajador, representando de esta manera una situación que puede romper el equilibrio físico, mental o social del trabajador, generando pérdidas o tiempos muertos en una organización o empresa. Por tal motivo, realizaron visitas a las instalaciones de las empresas de Guaya Fina, para conocer a través de la observación directa las diversas actividades ejecutadas, permitiendo identificar los riesgos a los que pueden incurrir los trabajadores en la ejecución de las operaciones que se realizan.

En este diagnóstico, presumen que se ejecutan bajo condiciones de riesgo, debido a que se desarrollan en pozos petroleros donde se presencia un gas tóxico como el sulfuro de hidrogeno (H2S). Asimismo, se observó falta de mantenimiento a los equipos, deficiencias de materiales, un medio ambiente inadecuado para el personal y los equipos que allí operan, lo que traería como consecuencia una organización deficiente.

En virtud de lo expuesto, y bajo una investigación de tipo descriptiva, con diseño de campo, no experimental y transeccional se estableció como propósito determinar los riesgos operacionales en las empresas de guaya fina en la Costa Oriental del Lago.

\section{MÉTODO}

Considerando el objetivo planteado para determinar los riesgos operacionales en las empresas de guaya fina en la Costa Oriental del Lago, esta investigación se considera de tipo descriptiva. En este orden, Arias (2006) explica que el tipo descriptiva, consiste en la caracterización de un hecho, fenómeno, individuo o grupo, con el fin de establecer su estructura o comportamiento.

De la misma manera, el estudio estuvo orientado hacia un diseño de campo, no experimental y transeccional. De campo, debido a que los datos fueron obtenidos de una situación real, a través de un instrumento de recolección de datos denominado cuestionario, al mismo tiempo, se orientó con un diseño no experimental, por cuanto no se manipuló deliberadamente la variable de estudio, sino que ésta se analizó en su contexto natural y finalmente se identificó bajo un diseño transeccional, ya que los datos se recolectaron en un solo momento, es decir en un tiempo único.

Por otra parte, el desarrollo de la investigación requiere el establecimiento de los parámetros dentro de los cuales operará el estudio. En razón de esto, la población quedó representada por las empresas que realizan operaciones de guaya fina en la Costa Oriental del Lago, cuyas unidades de información fueron gerentes generales, gerentes de operaciones y supervisores de seguridad, considerados como el personal vinculado a la gestión de riesgos en estas empresas para un total de 25 sujetos, enmarcándose en un censo poblacional. 
Desde esta perspectiva, para recolectar los datos que permitieron alcanzar el objetivo de investigación, se empleó como instrumento el cuestionario, con una escala de respuestas específica, la cual en el caso de la investigación es cerrada, dicotómica, con dos alternativas ( $\mathrm{Sl}$ y NO). Análogamente, el instrumento diseñado se sometió a un proceso de validación de contenido, a través de la técnica del juicio de expertos y para calcular la confiabilidad del instrumento de recolección de datos, se utilizó el método denominado Coeficiente de Küder - Richardson, el cual obtuvo un valor de 0,87.

Para el análisis estadístico de los datos se utilizó la estadística descriptiva, que permitió caracterizar cada uno de los indicadores, a través del uso de las frecuencias absolutas y relativas. Para la interpretación de las frecuencias relativas resultantes para se consideró establecer un baremo, el cual se muestra en la Tabla 1.

Tabla 1. Baremo para la interpretación de las frecuencias

\begin{tabular}{cc}
\hline \multicolumn{2}{c}{ RANGO PARA FRECUENCIA RELATIVA } \\
\hline $\mathrm{SI} \geq 70 \%$ & Alta presencia \\
$40 \% \leq \mathrm{SI}<70 \%$ & Mediana presencia \\
$\mathrm{SI}<40 \%$ & Baja presencia \\
\hline
\end{tabular}

Fuente: Elaboración propia

RESULTADOS

En la Tabla 2 se muestran las proporciones de respuestas para los ítems del indicador riesgos físicos. Se evidencia que, el $64 \%$ de los encuestados consideraron la opción afirmativa, indicando mediana presencia de los riesgos físicos en la gestión de riesgos que llevan a cabo las empresas estudiadas. Estos resultados están delineados por los niveles de presencia asignados a cada una de las actividades medidas por los reactivos aplicados para medir el indicador.

Tabla 2. Indicador: Riesgos físicos

\footnotetext{
ítems

1. ¿̇Han identificado los riesgos físicos a los cuales está expuesto el trabajador, previo al inicio de la ejecución de actividades?

2. ¿ ¿Brindan orientación a los trabajadores en la prevención de riesgos físicos?

3. ¿̇En las instalaciones de operaciones los riesgos físicos han sido controlados?
} 
4. $\quad$ ¿Los trabajadores sometidos a altos niveles de ruido en su puesto de trabajo son protegidos del mismo con los elementos de seguridad adecuados?

5. ¿Utilizan procedimientos administrativos para el control de las temperaturas extremas? (ejemplo: climatización al calor, régimen de trabajo, descanso diseñado para reducir los índices de estrés, distribución de la carga de trabajo y realización de estas en las horas frescas del día).

\begin{tabular}{|c|c|c|c|c|c|c|c|c|c|c|}
\hline \multicolumn{11}{|c|}{ Respuestas: Escala dicotómica } \\
\hline & \multicolumn{2}{|c|}{1} & \multicolumn{2}{|c|}{2} & \multicolumn{2}{|c|}{3} & \multicolumn{2}{|c|}{4} & \multicolumn{2}{|c|}{5} \\
\hline Opción & $\mathrm{Fa}$ & $\%$ & $\mathrm{Fa}$ & $\%$ & $\mathrm{Fa}$ & $\%$ & $\mathrm{Fa}$ & $\%$ & $\mathrm{Fa}$ & $\%$ \\
\hline $\mathrm{Si}$ & 22 & 88 & 18 & 72 & 15 & 60 & 19 & 76 & 6 & 24 \\
\hline No & 3 & 12 & 7 & 28 & 10 & 40 & 6 & 24 & 19 & 76 \\
\hline Total & 25 & 100 & 25 & 100 & 25 & 100 & 25 & 100 & 25 & 100 \\
\hline Categoría & \multicolumn{2}{|c|}{ Alta presencia } & \multicolumn{2}{|c|}{ Alta presencia } & \multicolumn{2}{|c|}{$\begin{array}{l}\text { Mediana } \\
\text { presencia }\end{array}$} & \multicolumn{2}{|c|}{ Alta presencia } & \multicolumn{2}{|c|}{$\begin{array}{c}\text { Baja } \\
\text { presencia }\end{array}$} \\
\hline Promedio & & & $\mathrm{Si}=64$ & & & & & $p=36 \%$ & & \\
\hline Categoría & \multicolumn{10}{|c|}{ Mediana presencia } \\
\hline
\end{tabular}

Fuente: Elaboración propia

Así entonces, se aprecia que a la hora de prevenir los riesgos físicos a los que están sometidos sus trabajadores, las siguientes actividades se toman con alta presencia: tienen identificados los riesgos físicos a los cuales está expuesto el trabajador, previo al inicio de la ejecución de actividades (88\%); brindan orientación a los trabajadores en la prevención de riesgos físicos (72\%); y los trabajadores sometidos a altos niveles de ruido en su puesto de trabajo son protegidos del mismo con los elementos de seguridad adecuados (76\%). Al mismo tiempo, catalogaron con mediana presencia el que en las instalaciones de operaciones los riesgos físicos hayan sido controlados (60\%). Sin embargo, denotaron con baja presencia lo referido a si utilizan procedimientos administrativos para el control de las temperaturas extremas (24\%).
Los resultados constatan mediana presencia, por ello, se deduce que hay mediana congruencia con lo expuesto por Burgos (2009), para quien los riesgos físicos son factores propios de las operaciones realizadas en el puesto de trabajo y alrededores, producto, generalmente, de las instalaciones y equipos. Estos riesgos incluyen ruidos, temperaturas extremas, presión, humedad, iluminación, vibración y electricidad. Dichos riesgos, deben ser considerados al momento de planificar las acciones preventivas en la gestión de riesgos, de manera que puedan ser minimizados sus efectos.

En esta perspectiva, los resultados obtenidos evidencian que en las empresas bajo análisis se realizan con mediana presencia las medidas preventivas para evitar los efectos que pudieran ocasionar los riesgos físicos en sus operaciones; por ello, a juicio de las investigadoras, en estas empresas no se 
toma como prioridad la prevención de este tipo de riesgo, obviando de alguna manera que estos riesgos, deben formar parte clave en la prevención de riesgos que planifique la gestión de seguridad de la empresa.

Seguidamente, en la Tabla 3, se muestran los valores obtenidos de las respuestas correspondientes a los ítems relacionados al indicador riesgos químicos. Se observa cómo, el $66 \%$ de los sujetos encuestados respondieron de manera afirmativa a las proposiciones emitidas, indicando que las actividades relacionadas se realizan con mediana presencia.

Al detallar estos resultados, se aprecia como para el $76 \%$ de los encuestados, han identificado los riesgos químicos a los cuales está expuesto el trabajador previo al inicio de la ejecución de actividades, y se brinda orientación a los trabajadores en la prevención de riesgos químicos, calificando estas dos actividades con alta presencia en la gestión de riesgos que ejecutan. No obstante, calificaron con mediana presencia el que: en las instalaciones de operaciones los riesgos químicos hayan sido controlados (64\%); brinden orientación a los trabajadores de los riesgos a los que se someten al manipular sustancias en forma gaseosa que normalmente se encuentran en estado líquido o sólido (64\%); y se apliquen medidas para prevenir las intoxicaciones ocupacionales producto de la manipulación de vapores (52\%).

Tabla 3. Indicador: Riesgos químicos

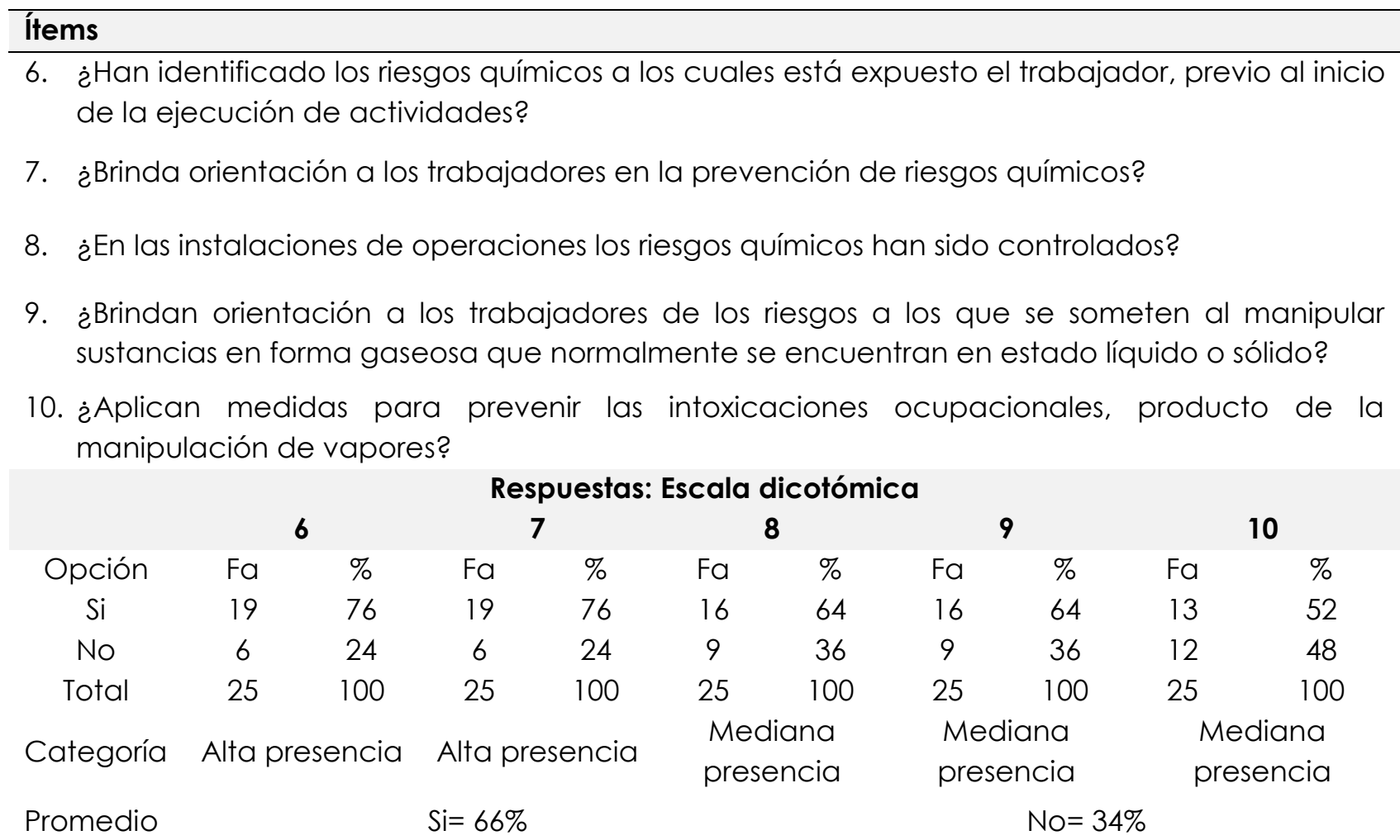


Fuente: Elaboración propia

Los resultados validan medianamente lo expuesto por Burgos (2009), para quien los riesgos químicos son aquellos riesgos a los que se somete el trabajador por exposición a sustancias químicas que se encuentran en las áreas de trabajo o en sus alrededores. Por ello, en cualquier gestión de riesgos, la prevención a estos factores es pieza clave, por lo cual se debe evitar la inhalación, ingestión y/o contacto con cualquiera de ellos y tomar las precauciones necesarias para la manipulación, ya que el uso, transporte - almacenamiento inadecuado de estos puede ocasionar consecuencias negativas o efectos adversos para la salud humana.

A juicio de las investigadoras, en las empresas analizadas, se está realizando de manera poco satisfactoria la prevención de los riesgos químicos, dado que todas las actividades medidas para

Tabla 4. Indicador: Riesgos biológicos su prevención se realizan con mediana frecuencia, obviando en todo caso que son medidas preventivas que deben considerarse cuando se está en presencia de estos riesgos.

En relación al indicador riesgos biológicos, la Tabla 4 muestra las respuestas correspondientes donde se evidencia que el $46 \%$ de los encuestados, en promedio, consideraron que en las empresas se tiene mediana presencia de las actividades relacionadas al indicador. Esta situación es explicada por el comportamiento de las respuestas afirmativas dadas a cada ítem involucrado, las cuales arriban todas a las categorías de mediana y baja presencia, indicando que en la prevención de los riesgos operacionales, los riesgos biológicos son considerados medianamente.

\section{Ítems}

11. ¿Han identificado los riesgos biológicos a los cuales está expuesto el trabajador antes de la ejecución de actividades?

12. ¿̇rindan orientación a los trabajadores en la prevención de riesgos biológicos?

13. ¿En las instalaciones de operaciones los riesgos biológicos han sido controlados?

14. ¿̇Realizan campañas de prevención de contaminantes biológicos (microorganismos, cultivos de células, endoparásitos humanos, entre otros)?

15. ¿Aplican acciones de control sobre agentes biológicos peligrosos, capaces de causar alteraciones en la salud humana?

\section{Respuestas: Escala dicotómica}

\begin{tabular}{cccccccccccc} 
& \multicolumn{2}{c}{$\mathbf{1 1}$} & \multicolumn{2}{c}{12} & \multicolumn{2}{c}{$\mathbf{1 3}$} & \multicolumn{2}{c}{14} & \multicolumn{2}{c}{15} \\
Opción & $\mathrm{Fa}$ & & $\%$ & $\mathrm{Fa}$ & $\%$ & $\mathrm{Fa}$ & $\%$ & $\mathrm{Fa}$ & $\%$ & $\mathrm{Fa}$ & $\%$ \\
$\mathrm{Si}$ & 15 & 60 & 15 & 60 & 12 & 48 & 6 & 24 & 9 & 36 \\
$\mathrm{No}$ & 10 & 40 & 10 & 40 & 13 & 52 & 19 & 76 & 16 & 64
\end{tabular}




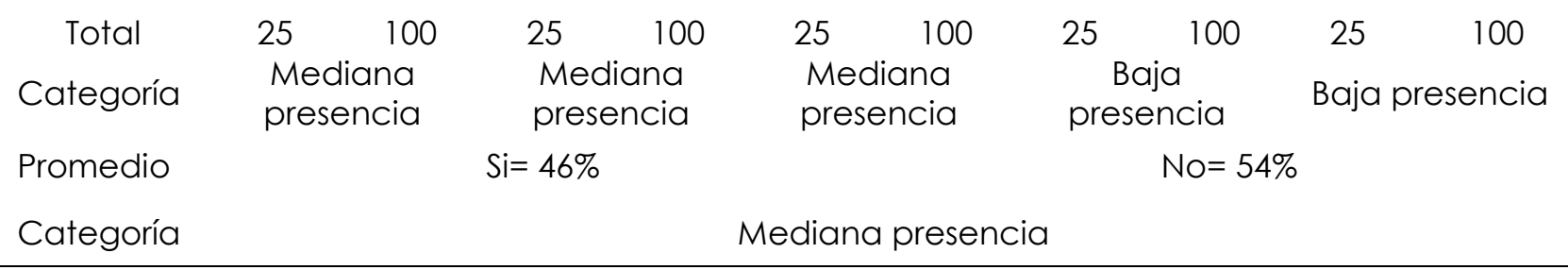

Fuente: Elaboración propia

De tal forma, en las empresas estudiadas se realizan con mediana frecuencia las actividades de prevención referidas a sí: han identificado los riesgos biológicos a los cuales está expuesto el trabajador antes de la ejecución de actividades (60\%); brindan orientación a los trabajadores en la prevención de riesgos biológicos (60\%); en las instalaciones de operaciones los riesgos biológicos hayan sido controlados (48\%). Y con baja presencia lo referido a si: realizan campañas de prevención de contaminantes biológicos (24\%), y apliquen acciones de control sobre agentes biológicos peligrosos capaces de causar alteraciones en la salud humana (36\%).

Los resultados validan medianamente a Pedreira (2009), para quien en la reducción de los riesgos de origen biológico, presentes en las organizaciones, la gerencia de riesgos debe identificar, evaluar y proponer correctivos que permitan controlar las condiciones y medio ambiente de trabajo que puedan afectar tanto la salud física como mental de los trabajadores y trabajadoras en el lugar de trabajo, o que pueden incidir en el ambiente externo del centro de trabajo o sobre la salud de su familia. Al mismo tiempo que deben brindar orientación a los trabajadores en la prevención de riesgos biológicos.

En este sentido, a criterio de las investigadoras, se confirma que en efecto el indicador riesgos biológicos no está siendo considerado como un riesgo clave a controlar dentro de la gestión de riesgos de las empresas bajo estudio, no comprendiendo que es menester de la gerencia de riesgos, a través de los servicios de seguridad en el trabajo, realizar 
inspecciones en los sitios de operaciones de la misma, conjuntamente con el comité de seguridad y salud laboral, con el propósito de identificar condiciones insalubres que puedan generar la presencia de contaminantes de orden biológicos, para establecer los controles pertinentes al caso y las mejoras inmediatas.

En el mismo orden de ideas, la Tabla 5 muestra las respuestas correspondientes al indicador riesgos disergonómicos, de la dimensión riesgos en las operaciones. Se aprecia que el $52 \%$ de los encuestados consideraron que, en las empresas bajo estudio, se dan con mediana presencia las actividades relacionadas a la prevención de estos riesgos.

Tabla 5. Indicador: Riesgos disergonómicos

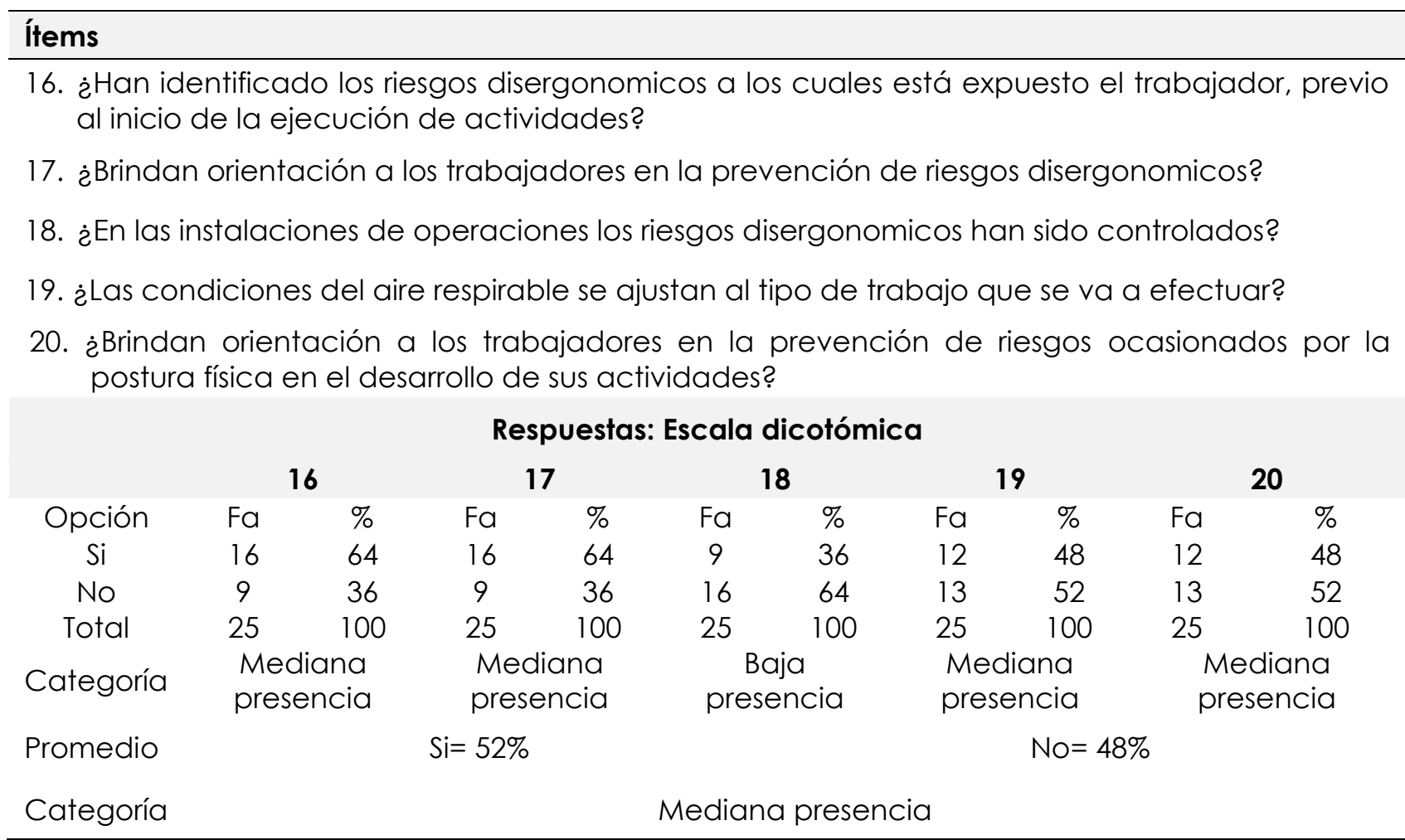

Fuente: Elaboración propia

De tal forma, se tiene mediana presencia de que: hayan identificado los riesgos disergonómicos a los cuales está expuesto el trabajador, previo al inicio de la ejecución de actividades (64\%); brinden orientación a los trabajadores en la prevención de riesgos disergonómicos (64\%); las condiciones del aire respirable se ajusten al tipo de trabajo que se va a efectuar (48\%); y brindan orientación a los trabajadores en la prevención de riesgos ocasionados por la postura física en el desarrollo de sus 
actividades (48\%). Y con baja presencia, han controlado en las

Los resultados evidenciados validan medianamente lo expuesto en la teoría que guía la investigación. Así, se logra cierta congruencia con lo postulado por Espeso (2009), quien establece que estos riesgos se generan cuando no se toman en cuenta las condiciones del sitio de trabajo, lo cual puede ocurrir entre los trabajadores que llevan a cabo tareas de mantenimiento en los equipos. Por ello, sus mayores intereses son el diseño del lugar de trabajo, posición en el trabajo, manejo de materiales, manuales, ciclos de trabajo/descanso y asientos.

A juicio de las investigadoras, la mediana presencia de este indicador indican que se están desaprovechando las oportunidades que ofrece el poseer una gestión de riesgos asertiva en la prevención de los mismos, donde los servicios de seguridad y salud en el trabajo deben tener como objetivos la promoción, prevención y vigilancia en materia de seguridad, salud, condiciones y medio ambiente de trabajo, para proteger los derechos humanos a la vida, salud e instalaciones de operaciones los riesgos disergonómicos (36\%).

integridad personal de los trabajadores, específicamente en la prevención de problemas generados por falta de espacios ergonómicos.

Continuando con el recorrido del análisis, la Tabla 6 presenta la frecuencia relativa de las respuestas correspondientes a los ítems del indicador riesgos psicosociales, evidenciándose que, en las empresas estudiadas, se da baja presencia a este tipo de riesgos en la prevención de los mismos dentro de la gestión de riesgos que llevan a cabo, arribando este indicador a una frecuencia de $34 \%$ de respuestas afirmativas, la cual indica baja presencia de las actividades medidas.

Los valores mostrados evidencian que, la actividad señalada en el ítem 25 es la única considerada con mediana presencia, el resto califica con baja presencia. Así, realiza con mediana frecuencia el que organicen programas de intervención para la prevención del estrés ocupacional (64\%). 
Tabla 6. Indicador: Riesgos psicosociales

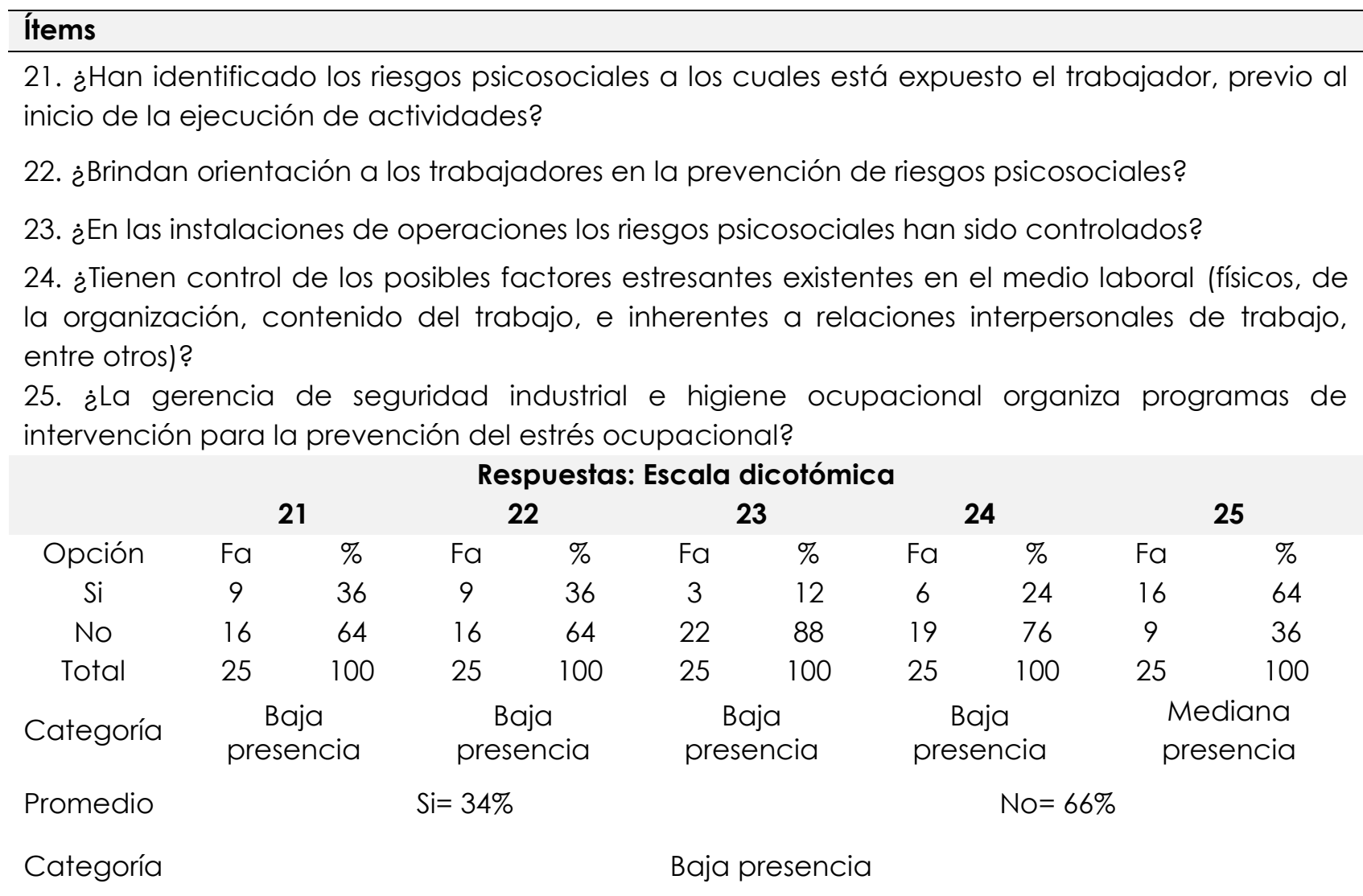

Fuente: Elaboración propia

Mientras, otorgaron baja presencia a lo concerniente a si: han identificado los riesgos psicosociales a los cuales está expuesto el trabajador, previo al inicio de la ejecución de actividades (36\%); brindan orientación a los trabajadores en la prevención de riesgos psicosociales (36\%); en las instalaciones de operaciones los riesgos psicosociales han sido controlados (12\%); y tienen control de los posibles factores estresantes existentes en el medio laboral (24\%).

Estos resultados, al reflejar baja presencia del indicador, pone de manifiesto baja coincidencia con lo expresado por Caldas, Castellanos, Hidalgo, y López (2009), quienes establecen que la exposición a unas condiciones psicosociales inadecuadas, puede tener efectos negativos en la 
salud de los trabajadores como el estrés, la insatisfacción laboral, la fatiga, el envejecimiento prematuro, el mobbing (acoso), entre otros. Por ello, se deben tomar las medidas preventivas pertinentes en toda gestión de riesgos que ejecute la gerencia de seguridad y salud ocupacional de la empresa.

A criterio de las investigadoras, esta situación mostrada evidencia que, en las empresas analizadas, se presta poco valor a los riesgos psicosociales dentro de la gestión de riesgos que ejecutan, no reconociéndolos como condiciones de la organización y gestión del trabajo, así como de las relaciones interpersonales tanto ocupacional como extra-ocupacional que dependiendo de la frecuencia, duración e intensidad pueden afectar la salud, seguridad, satisfacción y rendimiento laboral del trabajador. 
Finalmente, en cuanto a los riesgos operacionales, cuyos resultados se concentran en la Tabla 7, se observa cómo el 52\%, en promedio, de los encuestados consideraron la opción afirmativa, indicando mediana presencia de la prevención de los riesgos en las operaciones dentro la gestión de riesgos de las empresas de guaya fina en la Costa Oriental del Lago.

Tabla 7. Riesgos operacionales

\begin{tabular}{|c|c|c|c|}
\hline \multirow[t]{2}{*}{ INDICADORES } & \multicolumn{2}{|c|}{$\begin{array}{l}\text { RESPUESTAS / } \\
\text { ESCALA } \\
\text { DICOTÓMICA }\end{array}$} & \multirow[t]{2}{*}{ BAREMO } \\
\hline & $\begin{array}{c}\text { SI } \\
\operatorname{Fr}(\%)\end{array}$ & $\begin{array}{c}\text { NO } \\
\operatorname{Fr}(\%)\end{array}$ & \\
\hline Riesgos físicos & 64 & 36 & $\begin{array}{l}\text { Mediana } \\
\text { presencia }\end{array}$ \\
\hline Riesgos químicos & 66 & 34 & $\begin{array}{l}\text { Mediana } \\
\text { presencia }\end{array}$ \\
\hline $\begin{array}{l}\text { Riesgos } \\
\text { biológicos }\end{array}$ & 46 & 54 & $\begin{array}{l}\text { Mediana } \\
\text { presencia }\end{array}$ \\
\hline $\begin{array}{l}\text { Riesgos } \\
\text { disergonómicos }\end{array}$ & 52 & 48 & $\begin{array}{l}\text { Mediana } \\
\text { presencia }\end{array}$ \\
\hline $\begin{array}{l}\text { Riesgos } \\
\text { psicosociales }\end{array}$ & 34 & 66 & $\begin{array}{l}\text { Baja } \\
\text { presencia }\end{array}$ \\
\hline Total & 52 & 48 & $\begin{array}{l}\text { Mediana } \\
\text { presencia }\end{array}$ \\
\hline
\end{tabular}

Fuente: Elaboración propia

Al detalle, se puede apreciar que todos los tipos de riesgos, considerados como básicos en las operaciones de las empresas, arriban a la categoría de mediana presencia dentro de la prevención que debería implementar la gestión de riesgos de estas empresas (f́́sicos, químicos, biológicos y disergonómicos); agudizándose con baja presencia la prevención de los riesgos psicosociales.

Estos resultados validan de manera moderada la teoría expuesta por Cabaleiro (2010), para quien se deben conocer los tipos de riesgos en las operaciones para adoptar las medidas preventivas y eliminarles, minimizarlos 0 controlarlos, pues es esencial para evitar que se materialicen en un accidente laboral.

También alcanzan mediana congruencia con lo expresado por Hualde (2010), cuando afirma que, los riesgos operacionales son situaciones posibles que puedan reducir los ingresos de la empresa o puedan erosionar la imagen ocasionando una pérdida operacional futura, lo que es tratada como riesgo en las operaciones. Por ello, es importante reconocer que todas las actividades desarrolladas por la empresa conllevan a un riesgo operativo, el cual debe ser gestionado y medido para que el negocio se siga desarrollando de una manera óptima.

Para cerrar, las investigadoras observan que, en las empresas analizadas se da con mediana presencia las actividades propias de prevención de los riesgos operacionales en la gestión de riesgos que llevan a cabo, por lo cual están desestimando el valor implícito que conllevan las acciones preventivas al respecto.

De hecho, resulta prioritario actuar contra los riesgos que provocan accidentes o enfermedades, pero el 
término no se limita a ello, sino que hay que tener en cuenta todos los desequilibrios ocasionados a nivel de la salud, puesto que estos pueden afectar las diferentes actividades que se realizan en estas empresas de servicios petroleros, disminuyendo el rendimiento y la calidad de sus servicios.

\section{CONCLUSIONES}

$\mathrm{Se}$ determinaron los riesgos operacionales presentes en las empresas de guaya fina en la Costa Oriental del Lago, siendo ellos los riesgos: físicos, químicos, biológicos, disergonómicos y psicosociales. Cabe resaltar que, la prevención y control de estos riesgos están presentando debilidades para la organización, al develar los resultados mediana presencia en la gestión de riesgos analizada.

De manera más específica, se concluye que todos los tipos de riesgos operacionales asociados a la gestión de riesgos que se analizó, no están siendo considerados de manera acertada en la prevención de riesgos que realizan las empresas bajo estudio, dado que ostentaron mediana presencia, evidenciándose aún más en los riesgos psicosociales, que mostraron baja presencia en la prevención de los tipos estudiados.

En este caso se amerita la revisión, por parte de la gerencia, del cumplimiento de las actividades de prevención de los tipos de riesgos estudiados, como parte de su gestión de riesgos, garantizando la minimización de los efectos que pueden generar éstos. Al respecto estás empresas deben asumir conciencia de que las causas inmediatas de la ocurrencia de accidentes de trabajo es la falta de prevención oportuna de los actos inseguros y condiciones inseguras, y un efectivo control de estas causas permitirían reducir los accidentes o incidentes, de manera que una efectiva gestión de riesgos permitiría ejercer control coadyuvando a eliminar los accidentes.

\section{REFERENCIAS}

Arias, F. (2006). El proyecto de investigación. Introducción a la metodología científica. Cuarta edición. Editorial Episteme. CaracasVenezuela

Burgos, F. (2009). Salud industrial y ocupacional. Universidad de Carabobo. Trabajo de ascenso. Estado Carabobo. Venezuela

Cabaleiro, N. (2010). Prevención de riesgos laborales. Normativa de seguridad e higiene en el puesto de trabajo. Tercera edición. Editorial Vigo. España

Caldas, E.; Castellanos, A.; Hidalgo, M. y López, R. (2009). Formación y orientación laboral. Editorial Editex. Madrid. España

Espeso, M. (2009). Manual para la formación de técnicos de prevención de riesgos laborales. España: Editorial Lex Nova S.A 
Hualde, A. (2010). Análisis integral de riesgos operacionales y su aplicación en una empresa de ingeniería. Trabajo de grado para optar al título de Executive Master Financial Analysis. Universidad Carlos III. Madrid. España. Disponible en: ///http:worldwidescience.org/topicp ages/p/propuesta+legislativa+funda da.html

Menéndez, F. (2008). Higiene Industrial. Manual para la formación del especialista. Décima edición. Editorial LEX Nova. España

Pedreira, L. (2009). Tipos de riesgos laborales. Documento en línea. Disponible en: http://tiposderiesgoslaborales.blogsp ot.com/

Ruiz, C. (2007). Salud laboral, conceptos y técnicas para la prevención de riesgos laborales. Tercera edición. Editorial Mason. Barcelona. España 
